\title{
Structures and Thermochemistry of Calcium-Containing Molecules
}

\author{
Naomi L. Haworth, ${ }^{\llbracket}$ Michael B. Sullivan, ${ }^{\ddagger}$ Angela K. Wilson, ${ }^{\S}$ Jan M. L. Martin, ${ }^{\dagger}$ \\ and Leo Radom ${ }^{\top, t}$ \\ School of Chemistry, University of Sydney, Sydney, NSW 2006, Australia, Research \\ School of Chemistry, Australian National University, Canberra, ACT 0200, Australia, \\ Department of Chemistry, University of North Texas, Denton, Texas 76203-5070, USA, \\ and Department of Organic Chemistry, Weizmann Institute of Science, 76100 Rehovot, \\ Israel
}

\section{SUPPORTING INFORMATION}

(A total of 8 pages)

『 University of Sydney.

* Australian National Laboratory.

$\S$ University of North Texas.

$\dagger$ Weizmann Institute. 


\section{TABLE S1: Calculated Total Energies (Hartrees)}

\begin{tabular}{|c|c|c|c|c|c|c|c|c|c|c|}
\hline & \multirow[t]{2}{*}{$G 3^{a, b}$} & \multirow[t]{2}{*}{$\begin{array}{l}\mathrm{G} 3[\mathrm{CC}] \\
(\mathrm{dir}, \text { full) })^{, \mathrm{c}}\end{array}$} & \multicolumn{4}{|c|}{$\begin{array}{l}\operatorname{CCSD}(T) \\
\text { (aug-)cc-pWCVnZ }\end{array}$} & \multicolumn{4}{|c|}{$\begin{array}{l}\operatorname{CCSD}(T) \\
\text { (aug-)cc-pWCVn+xdZ }\end{array}$} \\
\hline & & & $\mathrm{TZ}$ & QZ & $5 Z$ & extrap. & $T+2 d Z$ & $Q+d Z$ & $5 Z$ & extrap. \\
\hline$\overline{\mathrm{Ca}}$ & -677.38326 & -677.36119 & -677.06211 & -677.09323 & -677.10207 & -677.11134 & -677.06739 & -677.09386 & -677.10207 & -677.11068 \\
\hline $\mathrm{H}$ & -0.50090 & -0.49982 & -0.49981 & -0.49995 & -0.49999 & -0.50002 & -0.49981 & -0.49995 & -0.49999 & -0.50002 \\
\hline $\mathrm{O}$ & -75.03029 & -75.01658 & -74.98223 & -74.99670 & -75.00129 & -75.00547 & -74.98223 & -74.99670 & -75.00129 & -75.00547 \\
\hline$F$ & -99.68259 & -99.66342 & -99.63295 & -99.65539 & -99.66270 & -99.66929 & -99.63295 & -99.65539 & -99.66270 & -99.66929 \\
\hline S & -397.95952 & -397.94680 & -397.65908 & -397.66896 & -397.67215 & -459.70631 & -397.65908 & -397.66896 & -397.67215 & -459.70631 \\
\hline $\mathrm{Cl}$ & -459.98877 & -459.97033 & -459.68052 & -459.69637 & -459.70136 & -397.67526 & -459.68052 & -459.69637 & -459.70136 & -397.67526 \\
\hline $\mathrm{CaH}$ & -677.94806 & -677.92279 & -677.62120 & -677.65800 & -677.66793 & -677.67818 & -677.62902 & -677.65881 & -677.66794 & -677.67740 \\
\hline $\mathrm{CaH}_{2}$ & -678.54547 & -678.51833 & -678.21848 & -678.25654 & -678.26691 & -678.27761 & -678.22603 & -678.25732 & -678.26691 & -678.27686 \\
\hline $\mathrm{CaO}$ & & -752.51727 & -752.16977 & -752.24040 & -752.25817 & -752.27572 & -752.19091 & -752.24203 & -752.25819 & -752.27445 \\
\hline $\mathrm{CaOH}$ & -753.23623 & -753.18969 & -752.85586 & -752.91257 & -752.92838 & -752.94415 & -752.86501 & -752.91342 & -752.92838 & -752.94334 \\
\hline $\mathrm{Ca}(\mathrm{OH})_{2}$ & -829.11228 & -829.04255 & -828.69212 & -828.79212 & -828.82226 & -828.85240 & -828.68745 & -828.75646 & -828.77769 & -828.79859 \\
\hline $\mathrm{CaF}$ & -777.26712 & -777.22017 & -776.88986 & -776.95216 & -776.97004 & -776.98762 & -776.89918 & -776.95302 & -776.97004 & -776.98681 \\
\hline $\mathrm{CaF}_{2}{ }^{\mathrm{f}}$ & -877.17188 & -877.10090 & -876.74288 & -876.83184 & -876.85800 & -876.88328 & -876.75274 & -876.83269 & -876.85801 & -876.88248 \\
\hline CaS & -1075.46831 & -1075.42288 & -1074.82464 & -1074.88340 & -1074.89922 & -1074.91531 & -1074.83846 & -1074.88461 & -1074.89923 & -1074.91429 \\
\hline $\mathrm{CaCl}$ & -1137.52786 & -1137.48184 & -1136.88883 & -1136.94441 & -1136.96021 & -1136.97641 & -1136.89678 & -1136.94521 & -1136.96022 & -1136.97564 \\
\hline $\mathrm{CaCl}_{2}$ & -1597.70259 & -1597.63329 & -1596.74864 & -1596.82584 & -1596.84815 & -1596.87089 & -1596.75748 & -1596.82667 & -1596.84815 & -1596.87010 \\
\hline $\begin{array}{ll}\text { a } & \text { Based } \\
\text { b } & \text { Includi } \\
\text { c } & \text { Not in } \\
\text { d } & \text { Based } \\
\text { e } & \text { Based }\end{array}$ & $\begin{array}{l}\text { on MP2(full)/6-3 } \\
\text { ng ZPVEs. } \\
\text { luding ZPVEs. } \\
\text { on CCSD(T)/(au } \\
\text { on CCSD(T)/(au }\end{array}$ & $\begin{array}{l}1 \mathrm{G}(d) \text { geometrie } \\
\text { g-)cc-pWCVQZ } \\
\text { g-)cc-pWCVQ+o }\end{array}$ & $\begin{array}{l}\text { s. } \\
\text { geometries. } \\
\text { Z geometries. }\end{array}$ & & & & & & & \\
\hline \multicolumn{11}{|c|}{$\begin{array}{l}\text { Single-point calculations were carried out using the linear geometries as the cc-pWCV } 5 Z \text { calculations were computationally too demanding to perform using a ben } \\
\text { configuration. }\end{array}$} \\
\hline
\end{tabular}


TABLE S2: Experimental Heats of Formation (kJ mol${ }^{-1}$ )

\begin{tabular}{|c|c|c|c|c|}
\hline & $\Delta_{\mathrm{f}} H^{0}{ }_{298}$ & Uncertainty & References $^{A}$ & Year $^{B}$ \\
\hline \multirow[t]{5}{*}{$\mathrm{CaH}$} & 229.4 & 2.0 & $b$ & 1994 \\
\hline & 230.7 & 1.4 & $f$ & 1983 \\
\hline & 233 & 7 & $g$ & 1983 \\
\hline & 229 & & c & 1982 \\
\hline & 229 & & $d, e$ & 1979 \\
\hline \multirow[t]{36}{*}{$\mathrm{CaO}$} & 43.9 & 21 & a & 1998(1964) \\
\hline & 62 & 19 & $\mathrm{a}, \mathrm{h}$ & 1998(1973) \\
\hline & 52 & & $a, i$ & 1998(1964) \\
\hline & 26 & & $a, i$ & 1998(1964) \\
\hline & 64 & & $a, i$ & 1998(1964) \\
\hline & 23 & & $a, j$ & 1998(1964) \\
\hline & 38.0 & 10 & $b$ & 1994 \\
\hline & 37 & 9 & $\mathrm{~b}, \mathrm{k}$ & 1994(1986) \\
\hline & 33 & 8 & $\mathrm{~b}, \mathrm{l}, \mathrm{m}$ & $1994(1984,1981)$ \\
\hline & 27 & 7 & $\mathrm{~b}, \mathrm{n}$ & 1994(1980) \\
\hline & 37 & 16 & $b, o$ & 1994(1976) \\
\hline & 42 & 7 & $b, o$ & 1994(1976) \\
\hline & 47 & 7 & $b, o$ & 1994(1976) \\
\hline & 10 & 20 & $\mathrm{~b}, \mathrm{~h}$ & 1994(1973) \\
\hline & 46 & 13 & $b, i$ & 1994(1964) \\
\hline & 19 & 35 & $b, j$ & 1994(1964) \\
\hline & 45 & & c & 1982 \\
\hline & 29 & 21 & $\mathrm{p}$ & 1981 \\
\hline & 46 & 16 & $q$ & 1981 \\
\hline & 27 & 7 & $\mathrm{n}$ & 1980 \\
\hline & 34 & & $d$ & 1979 \\
\hline & -24 & & $r$ & 1977 \\
\hline & -35 & 15 & $\mathrm{~s}$ & 1976 \\
\hline & 41.5 & 2.1 & 0 & 1976 \\
\hline & 48 & 8 & 0 & 1976 \\
\hline & 42.3 & 0.8 & 0 & 1976 \\
\hline & 42 & 8 & $\mathrm{t}$ & 1976 \\
\hline & 49 & 19 & $C$ & 1976(1973) \\
\hline & 26 & 21 & $D$ & 1976(1964) \\
\hline & 32 & 25 & $E$ & 1976(1964) \\
\hline & 43 & 13 & u & 1975 \\
\hline & 40.5 & 2.9 & $\mathrm{~h}$ & 1973 \\
\hline & 62 & 19 & $\mathrm{~h}$ & 1973 \\
\hline & 5 & & e & 1968 \\
\hline & 34 & 21 & $\mathrm{i}$ & 1964 \\
\hline & 70 & 25 & j & 1964 \\
\hline $\mathrm{CaOH}$ & -193.9 & 21 & a & 1998 \\
\hline
\end{tabular}




$\begin{array}{lccc}-196 & 13 & \text { a, v, F } & 1998(1972) \\ -230 & 21 & \text { a, w, F } & 1998(1968) \\ -173 & 15 & \text { b } & 1994 \\ -189 & 20 & \text { b, x, F } & 1994(1987) \\ -174 & 7 & \text { b, x, F } & 1994(1987) \\ -180 & 20 & \text { b, x, F } & 1994(1987) \\ -190 & 11 & \text { b, x, F } & 1994(1987) \\ -183 & 10 & \text { b, I, m, F } & 1994(1984) \\ -165 & 8 & \text { b, p, F } & 1994(1981) \\ -168 & 100 & \text { b, p, F } & 1994(1981) \\ -184 & 15 & \text { b, y, F } & 1994(1973) \\ -242 & & \text { b, w, F } & 1994(1968) \\ -222 & 10 & \text { b, w, F } & 1994(1968) \\ -190.8 & 8 & \text { x } & 1987 \\ -192.0 & 16 & \text { x } & 1987 \\ -192.6 & 8 & \text { x, F } & 1987 \\ -201 & & \text { c } & 1982 \\ -171 & 17 & \text { p } & 1981 \\ -186 & 6 & \text { r, F } & 1977 \\ -217 & 10 & \text { h, F } & 1973 \\ -209 & 7 & \text { h, F } & 1973 \\ -208 & 4 & \text { h, F } & 1973 \\ -190 & 13 & \text { y } & 1973 \\ -182 & & \text { v } & 1972 \\ -207 & & \text { z, F } & 1969 \\ -224 & & \text { w, F } & 1968 \\ -215 & & \text { w, aa, F } & 1968(1966) \\ -203 & 33 & \text { w, bb, F } & 1968(1965) \\ & & & \end{array}$

$\begin{array}{ccccc}\mathrm{Ca}(\mathrm{OH})_{2} & -611 & 38 & \text { a, F } & 1998 \\ & -635 & 21 & \text { a, w, F } & 1998(1968) \\ & -623 & 50 & \text { a, w, aa, F } & 1998(1966) \\ -615 & 84 & \text { a, w, bb, F } & 1998(1965) \\ & -598 & 15 & \text { b } & 1994 \\ -625 & 70 & \text { b, x, F } & 1994(1987) \\ & -598 & 15 & \text { b, x, F } & 1994(1987) \\ -620 & 11 & \text { b, cc, F } & 1994(1981) \\ -598 & 15 & \text { b, cc, F } & 1994(1981) \\ -609 & 20 & \text { b, r, F } & 1994(1977) \\ & -616 & 20 & \text { b, y, F } & 1994(1973) \\ -591 & 400 & \text { b, w, F } & 1994(1968) \\ -618 & 20 & \text { b, w, F } & 1994(1968) \\ & -605.8 & 8 & x & 1987 \\ -602.5 & 20 & \text { x } & 1987 \\ -609.5 & 8 & x, F & 1987 \\ -621 & & \text { dd, F } & 1977 \\ & -625 & 8 & \text { r, F } & 1977\end{array}$




\begin{tabular}{|c|c|c|c|c|}
\hline & -608 & 21 & $w, F$ & 1968 \\
\hline & -596 & 50 & w, aa, F & 1968(1966) \\
\hline & -587 & 84 & $w, b b, F$ & $1968(1965)$ \\
\hline & -544 & 50 & aa, $\mathrm{F}$ & 1966 \\
\hline & -597 & 84 & $\mathrm{bb}, \mathrm{F}$ & 1965 \\
\hline $\mathrm{CaF}$ & -272 & 8 & a & 1998 \\
\hline & -279 & 10 & a, ee & 1998(1968) \\
\hline & -275 & 13 & $a, f f$ & 1998(1966) \\
\hline & -274 & 8 & $a, g g$ & 1998(1965) \\
\hline & -307 & 29 & $a, h h$ & $1998(1964)$ \\
\hline & -270 & 7 & $a, \mathrm{ii}$ & 1998(1963) \\
\hline & -275 & 13 & $\mathrm{a}, \mathrm{ii}$ & 1998(1963) \\
\hline & -276.3 & 5.1 & $\mathrm{~b}$ & 1994 \\
\hline & -273.5 & 1.2 & $f$ & 1983 \\
\hline & -272 & & c & 1982 \\
\hline & -275 & & $d$ & 1979 \\
\hline & -278 & 8 & jj & 1978 \\
\hline & -278 & 12 & ee & 1968 \\
\hline & -278 & 10 & $G$ & 1968 \\
\hline & -275 & 11 & kk & 1968 \\
\hline & -278 & 6 & $\mathrm{H}$ & 1968(1963) \\
\hline & -268 & 11 & $\mathrm{ff}$ & 1966 \\
\hline & -268 & & gg & 1965 \\
\hline & -310 & 29 & hh & 1964 \\
\hline & -267 & 19 & ii & 1963 \\
\hline & -267 & 19 & ii & 1963 \\
\hline $\mathrm{CaF}_{2}$ & -784.5 & 8 & a & 1998 \\
\hline & -782 & 22 & a, II, J & $1998(1965)$ \\
\hline & -784 & 10 & $\mathrm{a}, \mathrm{II}, \mathrm{J}$ & $1998(1965)$ \\
\hline & -781 & 6 & $\mathrm{a}, \mathrm{mm}, \mathrm{J}$ & 1998(1965) \\
\hline & -809 & 17 & a, ii, J & 1998(1963) \\
\hline & -782 & 10 & $\mathrm{a}, \mathrm{ii}, \mathrm{J}$ & 1998(1963) \\
\hline & -788 & 8 & $\mathrm{a}, \mathrm{ii}, \mathrm{J}$ & 1998(1963) \\
\hline & -797 & 8 & $\mathrm{a}, \mathrm{nn}, \mathrm{J}$ & 1998(1963) \\
\hline & -791 & 7 & $\mathrm{a}, \mathrm{nn}, \mathrm{J}$ & 1998(1963) \\
\hline & -780 & 26 & $\mathrm{a}, \mathrm{\infty}, \mathrm{J}$ & $1998(1934)$ \\
\hline & -786 & 8 & $\mathrm{a}, \mathrm{\infty}, \mathrm{J}$ & $1998(1934)$ \\
\hline & -790.5 & 8 & $b$ & 1994 \\
\hline & -782 & 6 & c & 1982 \\
\hline & -729 & & $\mathrm{kk}$ & 1968 \\
\hline & -787 & 19 & $\mathrm{ff}$ & 1966 \\
\hline & -802 & & hh & 1964 \\
\hline & -794 & 8 & ii, J & 1963 \\
\hline CaS & 123.6 & 8 & a & 1998 \\
\hline
\end{tabular}




\begin{tabular}{|c|c|c|c|c|}
\hline & 124 & 7 & $a, j$ & $1998(1964)$ \\
\hline & 123 & 8 & $a, p p$ & 1998(1963) \\
\hline & 121.6 & 15 & b & 1994 \\
\hline & 118 & & $d$ & 1979 \\
\hline & 117 & 21 & $q q$ & 1974 \\
\hline & 134 & & $\mathrm{rr}$ & 1971 \\
\hline & 143 & 19 & j & 1964 \\
\hline & 119 & & $\mathrm{pp}$ & 1963 \\
\hline $\mathrm{CaCl}$ & -104.6 & 13 & $a$ & 1998 \\
\hline & -102 & & a, ss & 1998(1970) \\
\hline & -98 & & a, ss & 1998(1970) \\
\hline & -100 & & a, ss & 1998(1970) \\
\hline & -113 & & $\mathrm{a}, \mathrm{tt}$ & 1998(1969) \\
\hline & -95 & & a, uu & 1998(1969) \\
\hline & -97 & & $a, v v$ & 1998(1969) \\
\hline & -129 & 25 & $a, w w$ & $1998(1965)$ \\
\hline & -103.6 & 6 & b & 1994 \\
\hline & -97 & 10 & $b, y$ & 1994(1973) \\
\hline & -105 & 11 & $b, s s$ & 1994(1970) \\
\hline & -106 & 4 & b, ss & 1994(1970) \\
\hline & -104 & 17 & b, ss & 1994(1970) \\
\hline & -101 & 7 & $b, s s$ & 1994(1970) \\
\hline & -128 & 45 & $\mathrm{~b}, \mathrm{uu}, \mathrm{K}$ & 1994(1969) \\
\hline & -99.6 & 4 & $b, u u$ & 1994(1969) \\
\hline & -97.2 & 0.2 & $f$ & 1983 \\
\hline & -98 & & c & 1982 \\
\hline & -98 & & $d$ & 1979 \\
\hline & -97 & 7 & $y$ & 1973 \\
\hline & -99 & & v & 1972 \\
\hline & -97 & 13 & ss & 1970 \\
\hline & -104 & 13 & uu & 1969 \\
\hline & -99 & & uu, $\mathrm{K}$ & 1969 \\
\hline & -142 & 42 & e & 1968 \\
\hline & -130 & 25 & ww & 1965 \\
\hline $\mathrm{CaCl}_{2}$ & -471.5 & 4 & a & 1998 \\
\hline & -485 & & $x x, L$ & 1998(1970) \\
\hline & -482 & & $x x, L$ & $1998(1970)$ \\
\hline & -472 & & yy, L & $1998(1964)$ \\
\hline & -467 & & zz, L & 1998(1963) \\
\hline & -467 & & $\mathrm{ZZ}, \mathrm{M}$ & 1998(1963) \\
\hline & -476 & & aaa, L & 1998(1963) \\
\hline & -476 & & aaa, L & 1998(1963) \\
\hline & -476 & & aaa, L & 1998(1963) \\
\hline & -477 & & bbb, L & 1998(1963) \\
\hline & -498 & & Ccc, L & $1998(1922)$ \\
\hline & -484.8 & 7 & b & 1994 \\
\hline
\end{tabular}




$\begin{array}{lccc}-472 & & \text { c } & 1982 \\ -485 & 21 & \text { y } & 1973 \\ -481 & & \text { v } & 1972 \\ -461 & 8 & \text { ss } & 1970 \\ -440 & 22 & \text { ww } & 1965 \\ -475 & & \text { zz, L } & 1963 \\ -488 & & \text { aaa } & 1963\end{array}$

A Upper case indicates comments on how the numbers were generated. Lower case indicates literature references. a-e represent compendia of reviewed data.

$\mathrm{B}$ The date given is for the most recent review of the data; the dates of the original report and of earlier reviews are given in parentheses.

C $t$, reinterpretation of $h$.

D s, reinterpretation of $i$.

E $\quad s$, reinterpretation of $j$.

F Calculated using $\mathrm{D}_{0}(\mathrm{OH})=425.89 \mathrm{~kJ} \mathrm{~mol}^{-1}$ from Ruscic, B.; Feller, D.; Dixon, D. A.; Peterson, K. A.; Harding, L. B.; Asher, R. L.; Wagner, A. F. J. Phys. Chem. A 2001, 105, 1.

$G$ Reviewed value from ee.

$\mathrm{H}$ From ee, review of ii.

$\mathrm{J}$ Calculated using $\Delta_{\mathrm{f}} \mathrm{H}^{0}\left(\mathrm{CaF}_{2}, \mathrm{cr}\right)=-1225.9 \mathrm{~kJ} \mathrm{~mol}^{-1}$ from a.

K Calculated based upon Hildenbrand, D. L.; Murad, E.; Potter, N. D.; Theard, L. D.; Hall, W. F., U-318, Contract AF 49(638)-1397, Aeronautic Div. of Philco Corp, Newport Beach, California.

L Calculated using $\Delta_{\mathrm{f}} H^{0}\left(\mathrm{CaCl}_{2}, \mathrm{cr}\right)=-795.8 \mathrm{~kJ} \mathrm{~mol}^{-1}, H^{1045}-H^{298}(\mathrm{cr})=59.401 \mathrm{~kJ} \mathrm{~mol}^{-1}$, $\Delta_{\text {fus }} H^{1045}=28.543 \mathrm{~kJ} \mathrm{~mol}^{-1}$ and $H^{1045}-H^{298}($ liq $)=66.424 \mathrm{~kJ} \mathrm{~mol}^{-1}$, all from a.

M Calculated using $\Delta_{\mathrm{f}} H^{0}\left(\mathrm{CaCl}_{2}, \mathrm{cr}\right)=-795.8 \mathrm{~kJ} \mathrm{~mol}^{-1}$ from a.

a Chase, M. W., Jr. J. Phys. Chem. Ref. Data 1998, Monograph 9, 1.

b Gurvich, L. V.; Veyts, I. V.; Alcock, C. B. Thermodynamic Properties of Individual Substances.; CRC Press: Boca Raton, 1994; Vol. 3.

c Wagman, D. D.; Evans, W. H.; Parker, V. B.; Schumm, R. H.; Halow, I.; Bailey, S. M.; Churney, K. L.; Nuttall, R. L. J. Phys. Chem. Ref. Data, 1982, 11, Supplement 2.

d Huber, K. P.; Herzberg, G. Constants of diatomic molecules.; van Nostrand Reinhold Company: New York, 1979; Vol. IV.

e Gaydon, A. D. Dissociation energies and spectra of diatomic molecules; Chapman and Hall: London, 1968.

f Rao, T. V. R.; Reddy, R. R.; Reddy, A. S. J. Mol. Struct. (Theochem) 1983, 14, 249.

g Martin, H., private communication quoted in Pettersson, L. G. M.; Siegbahn, P. E. M.; Ismail, S. Chem. Phys. 1983, 82, 355.

h Kalff, P. J.; Alkemade, C. T. J. J. Chem. Phys. 1973, 59, 2572.

i Drowart, J.; Exsteen, G.; Verhaegen, G. Trans. Farad. Soc. 1964, 60, 1920.

j Colin, R.; Goldfinger, P.; Jeunehomme, M. Trans. Farad. Soc. 1964, 60, 306.

k Kazenas, E. K.; Samoilova, I. O. VINITI, Deposited Doc. No. 5149-86 1986.

I Belyaev, V. N.; Lebedeva, N. L.; Krasnov, K. S.; Gurvich, L. V. Teplofiz. Vysokikh Temperatur. 1984, 22, 1008.

m Belyaev, V. N.; Lebedeva, N. L.; Krasnov, K. S.; Gurvich, L. V. Khimiya i Khim. Tekhnologiya 1981, 24, 778.

n Irvin, J. A.; Dagdigian, P. J. J. Chem. Phys. 1980, 73, 176.

o $\quad$ Farber, M.; Srivastava, R. D. High Temp. Sci. 1976, 8, 73.

p Murad, E. J. Chem. Phys. 1981, 75, 4080.

q Belyaev, V. N.; Gurvich, L. V.; Lebedeva, N. L.; Krasnov, K. S. VINITI, Deposited Doc. No. 3479-81 1981, 34. 
r Starovoitov, E. M.; Ryabova, V. G.; Gurvich, L. V.; Khitrov, A. N.; Nazarenko, I. I.; Belyaev, A. V. High Temp. 1977, 15, 770.

s Engelke, F.; Sander, R. K.; Zare, R. N. J. Chem. Phys., 1976, 65, 1146

t Srivastava, R. D. High Temp. Sci., 1976, 8, 225.

u J. Phys. Chem. Ref. Data 1975, 4, 1.

v Ryabova, V. G.; Gurvich, L. V. High Temp. 1972, 10, 669.

w Cotton, D. H.; Jenkins, D. R. Trans. Farad. Soc. 1968, 64, 2988.

X Farber, M.; Srivastava, R. D.; Moyer, J. W.; Leeper, J. D. J. Chem. Soc., Faraday Trans. 1 1987, 83, 3229.

y Gurvich, L. V.; Ryabova, V. G.; Khitrov, A. N. Faraday Symp. Chem. Soc. 1973, 8, 83.

z Ryabova, V. G.; Gurvich, L. V. 16th Prikl. Spektrosk., Mater. Soveshch. 1969, 1, 258.

aa Sugden, T. M.; Schofield, K. Trans. Farad. Soc. 1966, 62, 566.

bb Ryabova, V. G.; Gurvich, L. V. High Temp. 1965, 3, 284.

cc Matsumoto, K.; Sata, T. Bull. Chem. Soc. Jpn. 1981, 53, 647.

dd Ryabova, V. G.; Gurvich, L. V.; Khitrov, A. N.; Nazarenko, I. I. 7th Vses. Konf. Kalorim., [Rasshir. Tezisy Dokl.] 1977, 2, 293.

ee Hastie, J. W.; Margrave, J. L. J. Chem. Eng. Data 1968, 13, 428.

ff Hildenbrand, D. L.; Murad, E. J. Chem. Phys. 1966, 44, 1524.

gg Hildenbrand, D. L.; Murad, E. J. Chem. Phys. 1965, 43, 1400.

hh Ryabova, V. G.; Gurvich, L. V. High Temp. 1964, 2, 749.

ii Blue, G. D.; Green, J. W.; Bautista, R. G.; Margrave, J. L. J. Phys. Chem. 1963, 67, 877.

jj Karny, Z.; Zare, R. N. J. Chem. Phys. 1978, 68, 3360.

kk Hildenbrand, D. L. J. Chem. Phys. 1968, 48, 3657.

II Freeman, R. D., Tech. Doc. Rept. ASD TDR 63-754 Part II, Oklahoma State University, 1965.

$\mathrm{mm}$ Pottie, R. W., quoted in II.

nn Schultz, D. A.; Searcy, A. W J. Phys. Chem 1963, 67, 103.

oo Ruff, O.; Le Boucher, L. Z. anorg. allgem. Chem. 1934, 219, 376.

pp Marquart, J. R.; Berkowitz, J. J. Chem. Phys. 1963, 39, 283.

qq Mills, K. C. Thermodynamic Data for Inorganic Sulphides, Selenides and Tellurides; Butterworths: London, 1974.

rr U. S. Natl. Bur. Stand. Tech. Note 270-6, 1971.

ss Hildenbrand, D. L. J. Chem. Phys. 1970, 52, 5751.

tt Potter, N. D., personal communication, 1969, quoted in a.

uu Zmbov, K. F. Chem. Phys. Lett. 1969, 4, 191.

vv Hildenbrand, D. L. CPIA Publication No. 189 1969, 1, 86.

ww Ryabova, V. G.; Gurvich, L. V. HighTemp., 1965, 3, 604.

xx Lukashenko, E. E.; Reutova, G. A. Zh. Fiz. Khim. 1970, 44, 600.

yy Novikov, G. I.; Gavryuchenkov, F. G. Zh. Neorg. Khim. 1964, 9, 475.

zz Bautista, R. G.; Margrave, J. L. J. Phys. Chem. 1963, 67, 2411.

aaa Hildenbrand, D. L.; Potter, N. D. J. Phys. Chem. 1963, 67, 2231.

bbb Dewing, E. W., quoted in aaa.

ccc von Wartenberg, H.; Bosse, O. Zeitschrift fuer Elektrochemie und Angewandte Physikalische Chemie 1922, 28, 384. 